\title{
The X-Ray Mirrors for the Astro-E2 Mission
}

\author{
Kai-Wing Chan", Yang Soong \\ Universities Space Research Association \& NASA/Goddard Space Flight Center \\ Peter J. Serlemitsos \\ Laboratory for High Energy Astrophysics \\ NASA/Goddard Space Flight Center, Greenbelt, MD 20771, USA
}

\begin{abstract}
The X-Ray telescopes (XRT) for the US/Japan collaborative mission Astro-E2 will be of the same basic design as those built for the original Astro-E mission which failed to reach orbit in Feb. 2000. The NASAVGSFC X-ray Astrophysics Branch will again provide the five lightweight, broad-band mirrors for the mission. X-ray calibrations of the mirrors delivered for the original Astro-E instrument showed spatial resolutions characterized by Half-Power Diameters (HPD) in the range of $1.8-2.2$ minutes of arc, essentially independent of photon energy in the soft $x$-ray band. For the mission Astro-E2, both funding constraints and management decisions drastically limit any design modifications, so reflector fabrication and assembly procedures have remained largely unchanged. Nevertheless, in view of the importance in scientific return of attaining even a modest improvement in the spatial resolution of these mirrors, we have carefully considered the various sources of spatial error and, whenever possible, incorporated promising modifications. In this paper, we discuss our current understanding of the various error components as well as the small changes we have been able to implement.
\end{abstract}

Keywords: X-Ray Optics, X-Ray Telescopes, Thin-Foil Mirrors, Astro-E2

\section{INTRODUCTION}

The X-Ray Telescopes for the mission Astro-E2 will be of the same basic design as those built for the original Astro-E mission ${ }^{1}$. Each of these telescopes consists of circularly nested thin foil reflectors with metallic (gold or platinum) surfaces, focusing soft $(<10 \mathrm{keV}) \mathrm{x}$-rays at grazing angles in two reflections. For four of these telescopes, XRT-I, having focal lengths of $4.75 \mathrm{~m}$, the focal plane detectors are charge-coupled devices (CCD). The fifth telescope, XRT-S, with a focal length of $4.50 \mathrm{~m}$, will be used with the prime instrument of the mission, a microcalorimeter detector for high resolution spectroscopy. The basic parameters of the Astro-E2 XRT are listed in Table 1.

$X$-ray calibrations of the mirrors delivered for the original Astro-E instrument showed point spread functions (PSF) in the range of 1.8-2.2 minutes of arc $\mathrm{HPD}^{2}$. In this paper, we report on the measurements and analyses of the major error terms limiting the angular resolution of these telescopes, as well as on the various effects that impact their imaging quality. Preliminary x-ray measurements of sets of reflectors in quadrant housings, sampling the entire range of reflector

\footnotetext{
"Contact author at: kwchan@milkyway.gsfc.nasa.gov; 1-301-286-6238; Laboratory for High Energy Astrophysics, NASA/Goddard Space Flight Center, Code 662, MD 20771, USA.
} 
radii, show PSFs in the range $1.2-1.7$ arc-minute HPD. Repeatability of $x$-ray measurements for a particular set and conditions is 0.04 arc minute. An example of the image, measured as photon counts on a focal plane CCD is shown in Figure 1.

\begin{tabular}{|l|c|c|c|}
\hline Parameters & Unit & XRT-I & XRT-S \\
\hline Focal Length & $\mathrm{mm}$ & 4750 & 4500 \\
\hline Substrate & & Epoxy on Al & Epoxy on Al \\
\hline Reflecting Surface & & Gold & Platinum \\
\hline Al Substrate Thickness & $\mu \mathrm{m}$ & 155 & 155 \\
\hline Epoxy thickness & $\mu \mathrm{m}$ & 13 & 13 \\
\hline Number of Shell & & 175 & 168 \\
\hline Segment per shell & & 4 & 4 \\
\hline Mirror Dimension & & & 57.9 \\
\hline Inner Radius (@ Sec. Bot.) & $\mathrm{mm}$ & 57.9 & 200.0 \\
\hline Outer Radius (@ Prim. Top) & $\mathrm{mm}$ & 199.5 & 101.6 \\
\hline Mirror Length & $\mathrm{mm}$ & 101.6 & 11.1 \\
\hline Gap between Prim. \& Sec. & $\mathrm{mm}$ & 11.1 & 0.188 \\
\hline Inner grazing angle & degree & 0.178 & 0.634 \\
\hline Outer grazing angle & degree & 0.599 & \\
\hline
\end{tabular}

Table 1. Basic Parameters of Astro-E2 X-Ray Telescopes.
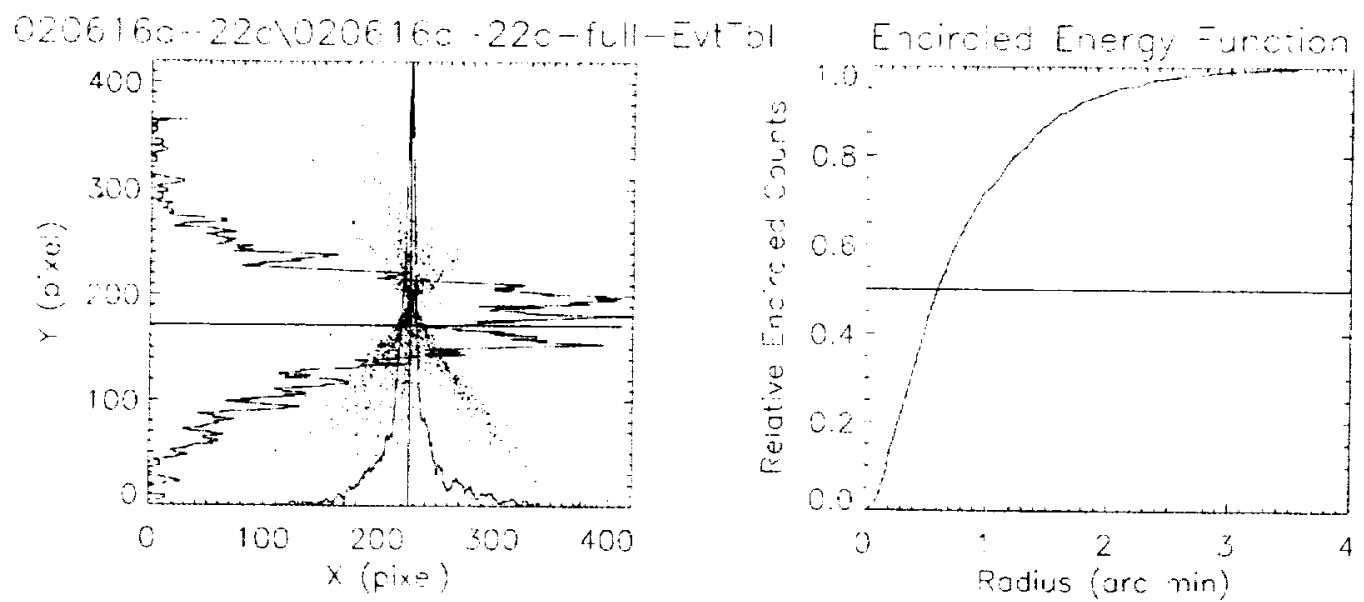

Figure. 1. CCD image of the mirror test module and the inferred half-power radius from the corresponding encircled energy function.

In the following, we discuss three major components of spatial error: axial figure errors of individual reflectors, radial alignment errors of groups of reflectors of different radii, and the dispersion of focal centroids of quadrant sectors. We also discuss point spread function dependence on mirror temperature and on quadrant orientation under the influence of gravity. Although gravity plays no role on the space borne instrument, it does introduce ambiguity in ground calibrations and alignments, especially with the unsecured thin reflectors we use to assemble our mirrors. 


\section{AXIAL FIGURE ERRORS}

In the conical approximation of the Wolter Type I mirror design ${ }^{3}$, our foil reflectors begin as flat aluminum substrates, formed, under pressure and elevated temperature, into conical segments, approximately obeying the desired condition between curvature and cone angle. Smooth metallic surfaces are subsequently imparted on them via epoxy replication using metal-coated, pyrex glass mandrels ${ }^{4}$. These commercially available mandrels, affordable but unfortunately cylindrical by necessity, have good surface micro-roughness $(0.4-0.7 \mathrm{~nm} \mathrm{rms}$ over $10 \mu \mathrm{m}$ to $1 \mathrm{~mm}$ range). Aside from the basic figure mismatch (i.e. cylinder vs. cone), however, surface distortions and curvature are commonplace. Lacking any control over the supply of these glass tubes, we select a small fraction of those tested, discarding the remaining. Even those deemed as suitable suffer from as much as $4 \mu \mathrm{m}$ deviation from the desired straight profile over the $10 \mathrm{~cm}$ width of our reflectors. Since essentially all of our mandrels have residual curvature, we have made sure that we properly bias our selections to favor a concave mirror shape which is more in tune with the exact Wolter I geometry.

Obviously, excess curvature, even when concave, degrades performance just as badly.

In the conical approximation, the radius-dependent image size (traditionally quoted in terms of the half-power diameter) is $\approx a \alpha / 2 f$, where $a$ is the foil axial length, $\alpha$ is the grazing angle of reflection, and $f$ is the focal length of the telescope. To improve on the conical approach, the next level of approximation is to have a mirror with a concave axial curvature of which the depth deviates from a straight profile by an amount of $\approx a^{2} \alpha / 16 f$ (the exact magnitude depends on the actual axial profile.) The rate of image degradation with respect to the profile root-mean-square ( $\mathrm{rms}$ ) goes simply as $24 / a$ for low frequency profile variations, which is 0.8 arc-minute $/ \mu \mathrm{m}$. Simulation for $2^{\text {nd }}$ and $3^{\text {rd }}$ polynomial axial profiles confirms this rate of degradation. With the selection criterion of $4 \mu \mathrm{m}$ deviation peak-to-peak in axial curvature of replication mandrels, or equivalently $1.3 \mu \mathrm{m}$ rms for low frequency profiles, PSF degradation is expected to be limited to 1.0 arc minute HPD.

Using a laser-scanning micrometer, linear profile measurements were made of the full axial length on a set of 24 Astro-E2 reflectors. The sample included both primary and secondary foils, with radii distributed throughout the mirror range. Measurement repeatability and positioning accuracy were 0.2 and $0.1 \mu \mathrm{m}$ respectively. The measured profiles were smoothed with a $4 \mathrm{~mm}$ window in order to minimize extraneous slope error due to measurement noise $(0.2 \mu \mathrm{m}$ at a sampling step of $200 \mu \mathrm{m})$ but still keeping mm-scale features on the profile. Angular spread of rays from single specular reflection is derived from the slopes of the profiles. The results are summarized in Table 2 below.

\begin{tabular}{|l|l|l|}
\hline & Mean Value & Standard Deviation \\
\hline Profile rms $(\mu \mathrm{m})$ & $0.72+/-0.06$ & 0.31 \\
\hline Profile Amplitude $(\mu \mathrm{m})$ & $3.79+/-0.30$ & 1.48 \\
\hline Derived HPD $($ arc minute $)$ & $0.71+/-0.05$ & 0.22 \\
\hline
\end{tabular}

Table 2. Linear axial profile measurements of a set of 24 Astro-E2 reflectors.

These results are in line with the estimate of residual mandrel curvature. At 1- $\sigma$ statistical significance, the axial error is indeed expected to be $<1$ minute of arc. It is important to note 
however that, with an average blur from a single reflection of 0.7 arc-minutes HPD, the axial figure profile is the most significant error source to our measured mirror PSF.

\section{RANDOM RADIAL ALIGNMENT ERRORS}

In the mirror housing, reflectors are constrained and gang tuned using 4 sets of alignment bars or radial struts, made of aluminum, with grooves cut at proper positions. The Astro-E grooves or teeth were cut in a simple wedge shape, which meant that the very edges of reflectors were used for positioning the foils. Uniformity in reflector edges has always been a difficult problem for us so it is in this area where we introduced one of our minor design changes by incorporating a widened undercut at the bottom of the groove to nudge the contact point between foil and groove slightly inward in the foil and away from the edge. Even so, the modification resulted in only a partial solution to this problem.

The basic parameters of the alignment bars are listed in Table 3 . There are 13 alignment bars on each side of a primary and secondary quadrant housings, thus dividing a telescope quadrant into 12 angular regions or sectors. Half sectors on either side of the quadrant assembly are blocked.

\begin{tabular}{|l|c|c|}
\hline Parameters & Unit & \\
\hline Bar /Quadrant/Face & & 13 \\
\hline Quadrant & & 4 \\
\hline Face & & 4 \\
\hline Tooth /bar (XIS / XRS) & & $175 / 168$ \\
\hline Tooth Dimension & & \\
\hline Tooth width @ groove bot. & $\mu \mathrm{m}$ & 203 \\
\hline Tooth width @ groove top & $\mu \mathrm{m}$ & 363 \\
\hline Groove Height & $\mathrm{mm}$ & 1.52 \\
\hline Undercut depth & $\mu \mathrm{m}$ & 203 \\
\hline Width @ undercut neck & $\mu \mathrm{m}$ & 224 \\
\hline Tooth wall slant angle & degree & 3 \\
\hline Bar thickness & $\mathrm{mm}$ & 1.57 \\
\hline
\end{tabular}

Table 3. Basic design parameters for alignment bars of Astro-E2 XRT.

Tolerances in alignment groove positions, imperfection of foil edges where foils come into contact with groove boundaries and excess groove width in relation to the foil thickness, all contribute to the random alignment error of foil reflectors ${ }^{5}$. To assess the image quality at this level, $x$-ray measurements were made in a series of tests on our $\sim 40 \mathrm{~m}$ beam line, using 6-10 pairs of foils in quadrant housings, $4.5 \mathrm{keV} \mathrm{x}$-rays and a focal plane CCD. The areaweighed average blur for sectors was measured at 1.0-1.4 arc-minutes HPD. As discussed earlier, Astro-E2 reflectors in single reflection give an average blur of 0.7 arc-minute HPD which, for two uncorrelated reflections, increases in quadrature to 1.0 arc-minute. This is the expected axial figure error contribution to the above measurement. Additional errors included in the measurement come from a variety of sources including systematic offsets in alignment bar positioning, random errors associated with groove tolerances, reflector edge imperfections etc. The exact magnitudes of these additional slope errors are not modeled 
here. All of them are typically associated with radial errors of $5 \mu \mathrm{m}$ or more, corresponding to ray deflections estimated at 0.34 arc-minutes.

\section{SECTORAL POSITIONING ERRORS}

Rays from the 12 sectors combine to give the full image. Sector misalignments manifest themselves at the focal plane as a dispersion in sector centroids. Roundness error of individual reflectors as well as global distortions of the lightweight mirror housings contribute to this misalignment. X-ray testing gave the rms dispersion in sector centroids at $0.3-0.45$ arc-minutes. An example of the distribution of the sector centroids is shown in Figure 2 .

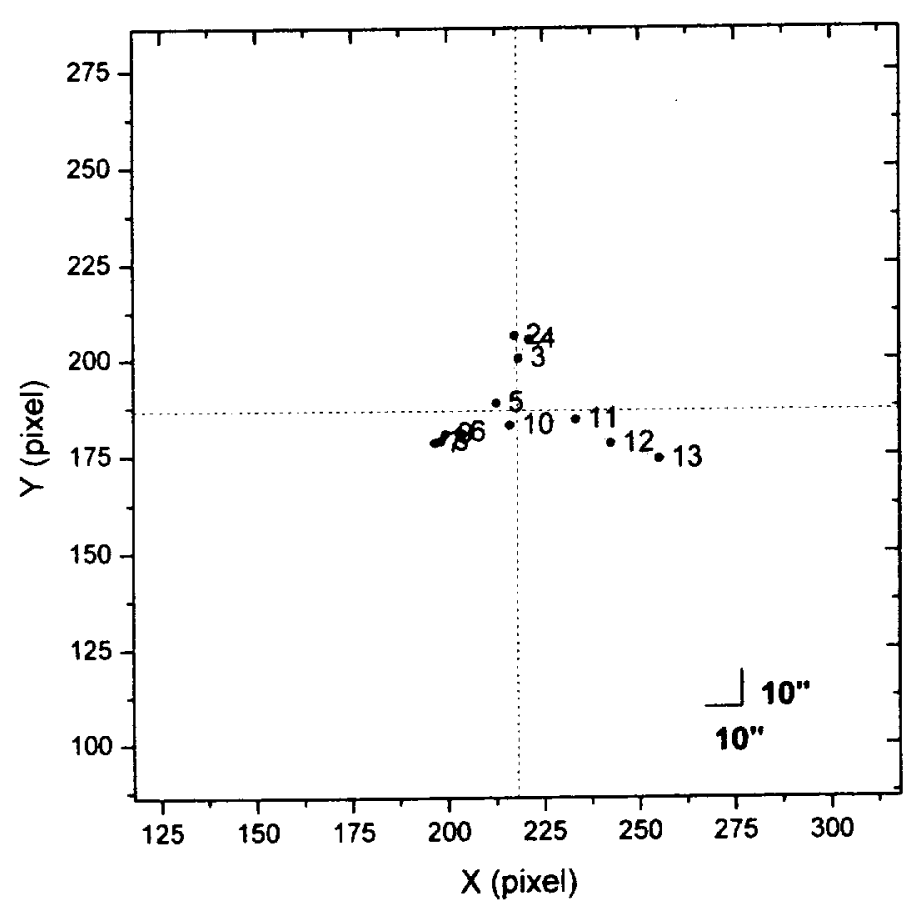

Figure 2. Variation of sector centroids on the focal plane.

As a rule of thumb, the overall image blur can be determined as a combination of sectoraveraged HPD $(S)$ and sector rms center variation $(C): H P D^{2}=S^{2}+(\xi C)^{2}$, where $\xi$ is a coefficient relating diameter and rms dispersion measures, which is dependent of the actual distribution of the photon on the focal plane. For most common distributions, $\xi$ is typically between 1 and 2. Empirically, $\xi=1.5$ is determined from calibration runs. From the measured values of sector-averaged HPD and centroid dispersion, it is seen that the sector misalignment, although not negligible, is not as significant an error as the axial figure error. As pointed out earlier, typically, $\mathrm{C}=0.4$. This magnitude of sector misalignment causes additional image degradation causing, for example, an otherwise average $S=1.2$ image to become one with 1.34 minutes HPD. 


\section{THERMAL EFFECTS ON IMAGE}

Each Astro-E2 XRT reflector consists of an aluminum substrate, a $\sim 1000 \AA$ thick metallic reflecting surface and an epoxy layer which bonds the tenuous reflecting surface onto the substrate. As such, a "bi-metallic" effect due to the different thermal expansion coefficients (CTE) between the two structural elements, aluminum and epoxy, can be expected.

Temperature induced stresses will alter the overall geometry of the reflectors and hence the angular response of the telescopes. Generally, the reflectors open up with elevated temperatures, since the CTE for the epoxy is greater than that for the aluminum.

The operational temperature of the Astro-E2 spacecraft is set as $12.5^{\circ} \mathrm{C}-27.5^{\circ} \mathrm{C}$. The temperature of the XRT is maintained by a set of heaters attached to the XRT housings. The heater controls are set to turn on and off at $15^{\circ} \mathrm{C}$ and $16^{\circ} \mathrm{C}$, respectively. To measure the potential degradation, we again used a small set of reflectors, characterized and assembled into a housing at room temperature $\left(-22^{\circ} \mathrm{C}\right)$. We then measured the PSF of the assembly in the range $10^{\circ} \mathrm{C}$ to $34^{\circ} \mathrm{C}$. We determined that the image degraded roughly at a rate of 0.009 $+/-0.002$ arc-minutes (HPD) $/{ }^{\circ} \mathrm{C}$. The degradation was similar on either side of room temperature. Thus, at the specified low limit of the spacecraft thermal environment, the reflectors are subjected to $\mathrm{a}-10^{\circ} \mathrm{C}$ change from the assembly environment, so the expected degradation of $\sim 0.1$ arc minute HPD is acceptably small. It should be noted that there may be small complications to this estimate due to a anisotropic thermal environment and the potential temperature gradients in mirrors it may produce, to inefficient heat transfer of the mirror heating elements etc.

\section{ORIENTATION EFFECTS}

One of the more subtle effects on our XRT evaluations is the "orientation effect", which is the dependence of the image quality on the orientation of the quadrant. Part of the problem is due to gravity induced foil distortions but an even more significant contribution is due to the fact that reflectors have not been secured to their supports and are thus free to move under gravity. Constraints imposed on us, as well as the short schedule of the Astro-E2 mission, have precluded any effort to abandon our long held free-reflector approach by securing reflectors in the housing. Alternatively, we have made every effort to reduce clearance $(\sim 10$ $\mu \mathrm{m})$ while, at the same time, avoiding reflector "pinching" which causes distortions. This scheme relies on uniform reflector thickness, so a separate effort became necessary to improve that condition. Nevertheless, significant reflector movement with orientation is still possible. Our calibrations on the beam line indeed show typically 0.2 arc-minute HPD variations depending on the orientation of the quadrant. It is quite difficult to know which orientation (if any) is more relevant to the gravity-free environment. A vertical $\mathrm{X}$-ray beam may well be the most appropriate for our situation but, at this stage, it is not available to us.

\section{QUADRANT ASSEMBLY}

Slope error in overall alignment or relative tilt between primary and secondary housing degrades the spatial resolution and shifts the focal distance from its nominal value. Much of our inability to maintain the proper alignment between the two quadrant halves is due to the flimsiness of the lightweight housing we are using. How to properly assemble the quadrant 
has been one of our major concerns since the start of the Astro-E2 project. It has become quite obvious that the direct mating of housing flanges results in housing distortions, which are subsequently transferred onto the previously aligned reflectors. To minimize this, we have been using a 3-point contact between the two housing halves. Because such an attachment will not pass the required structural qualification for flight hardware, we have additionally devised a procedure for applying epoxy shims, as needed to prevent distortion when torquing the remaining flange screws.

Image quality degrades with deviation of sensor location from the actual focal plane. For a medium size reflector of radius $11.5 \mathrm{~cm}$, the rate of degradation, from numerical simulations, is expected to be 0.12 arc-minute/cm. The scale is roughly proportional to the radius of the reflector. The weighed value for the Astro-E2 XRT is 0.14 arc-minute/cm (there is only a small difference between XRT-I and XRT-S). In an experiment, the variations were measured and the rates were found to be $0.16+/-0.013 \mathrm{arc}-$ minute $/ \mathrm{cm}$ and $0.09+/-0.02$ arcminute/cm (errors are statistical only), for the long and short side of the focal distance, respectively. The asymmetry may relate to the actual shape of the image in the measurement. The tolerance of focal length for the Astro-E2 XRT is broadly specified to be $2.5 \mathrm{~cm}(\approx 0.5 \%$ of focal length for these systems.) We will obviously make every effort to narrow this down. Based on our current state of the art, we believe that a goal of $\sim 1 \mathrm{~cm}$ is not out of the question.

\section{SUMMARY}

We have discussed the major error terms limiting the angular resolution of the Astro-E2 mirrors. In particular, the axial figure and the radial alignment errors are seen to be the dominant ones. Much of the axial error is due to the less than ideal but affordable replication mandrels we have settled for. At the start of the "rebuild" effort, we did somewhat tighten mandrel selection among those available to us from the original program. We have also briefly touched on the difficulties encountered as a result of the lightweight mirror housings mandated by the limited resources of the mission. Reflector alignment errors are coupled with gravity induced PSF changes, as discussed in the paper. The eventual impact of such effects in a gravity-free environment is difficult to evaluate. Temperature PSF dependence within the operational range of the instrument is not negligible but small.

We note that all discussion in this paper has been at the "quadrant level". PSF impacts from the misalignment of the 4 quadrants in the telescope assembly are relatively small. For example, a $100 \mu \mathrm{m}$ lateral shift in quadrant assembly amounts to 0.07 arc-minutes error.

As of July 2002 , more than 2500 reflectors, nearly enough for two full telescopes, have been produced. The foil production rate is progressing healthily at a pace sufficient to fill one telescope every 4 months. The assembly of the first telescope will start later in 2002 . All the telescopes are to be tested and delivered by early 2004 . 


\section{ACKNOWLEDGMENTS}

The authors would like to thank Dale Arbogast, Henry Garrett, Melinda Hong, John Kearney, Larry Lozipone, Larry Olsen and Marton Sharpe for their technical support, and Curtis Odell for his managerial support. This work is funded by NASA partly through the CPSS program at USRA.

\section{REFERENCES}

1. H.Kunidea, M.Ishida, T.Endo, Y.Hidaka, H.Honda, K.Imamura, J.Ishida, M.Maeda, K.Misaki, R.Shibata, A.Furuzawa, K.Haga, Y.Ogasaka, T.Okajima, Y.Tawara, Y.Terashima, M.Watanabe, K.Yamashita, T.Yoshioka, P.J.Serlemitsos, Y.Soong, K.W.Chan, "X-ray telescope onboard Astro-E: optical design and fabrication of thin foil mirrors", Appl. Opt. 40 (4), 553-564, 2001.

2. R. Shibata, M.Ishida, H.Kunieda, T.Endo, H.Honda, K.Misaka, J. Ishida,K.Imamura, Y.Hidaka,M.Maeda, Y.Tawara, Y.Ogasaka, A.Furuzawa, M.Watanabe, Y.Terashima, T.Yoshioka, T.Okajima, K.Yamashita, P.Serlemitsos, Y.Soong, K.W.Chan, "X-ray telescope onboard Astro-E. II. Ground-based x-ray characterization", Appl. Opt. 40 (22), 3762-3783, 2001.

3. P.J.Serlemitsos, Y.Soong, "Foil X-ray Mirrors", Astrophys. Sp. Sci., 239 (2): 177-196, 1996.

4. Y.Soong, L.Jolota, P.J.Serlemitsos, "Conical thin foil $\mathrm{x}$-ray mirror fabrication via surface replication", X-Ray and Extreme Ultraviolet Optics. Eds. R.B.Hoover, A.B.Walker. Proc. SPIE, 2515, 64-69, 1995.

5. Y. Soong, K.W.Chan, P.J.Serlemitsos, "Recent advance in segmented thin-foil X-ray optics", in X-Ray OPtics for Astronomy: Telescopes, Multilayers, Spectrometers, and Missions. Eds. P.Gorenstein and R.B.Hoover, Proc. SPIE, 4496, 54-61, 2001 